\title{
The Use of Natural Interaction to Enrich the User Experience in Telemedicine Systems
}

\author{
Tatiana A. Tavares ${ }^{1,2,3}$, Anna Medeiros ${ }^{3}$, Rafael de Castro ${ }^{3}$, and Eudisley dos Anjos ${ }^{1,2}$ \\ ${ }^{1} \mathrm{CI}$ - Informatics Institute, Campus Castelo Branco, s/n, \\ 58059-900 João Pessoa-PB, Brazil \\ ${ }^{2}$ LAVID - Digital Video Applications Lab, Campus Castelo Branco, s/n, \\ 58059-900 João Pessoa-PB, Brazil \\ ${ }^{3}$ PPGI - Informatics Graduate Program, Campus Castelo Branco, s/n, \\ 58059-900 João Pessoa-PB, Brazil \\ tatiana@lavid.ufpb.br, \\ \{linnamedeiros, rafaelhenriquec, guido\} @gmail.com, \\ eudisley@ci.ufpb.br
}

\begin{abstract}
Human communication always used gestures, movements and expressions as oral language support. Certain gestures are so commonly used around the world that are understood throughout different cultures and times, such as a wave or thumbs up. Natural Interaction is a way to apply this concept to user interfaces in computer systems. In this paper we discuss about the use of Natural Interaction features in a telemedicine system. More specifically, we demonstrate the use of natural interaction interfaces for control and manipulation of 3D objects inside Arthron tool. Arthron is a telemedicine tool used for surgery transmissions.
\end{abstract}

Keywords: Human Computer Interaction, Natural Interaction, Telemedicine.

\section{$1 \quad$ Introduction}

Making a brief retrospect we can identify the evolution of these devices through the command-based languages, through the graphical user interfaces (GUIs), and finally the direct manipulation with the advent of the use of the pointers (mouse). From the rise of touch screens, the use of cameras in the analysis of user actions and the creation of devices that allow us to use technology more easily. For example, the Kinect that enables us to think about the development of more sophisticated and natural user interfaces. The Natural Interaction (NI) studies ways that humans can interact through humans five senses, be that with gestures, voice commands, corporal expressions or human body parts detection and identification [1,2,3,4].

Natural User Interface or NUI is the used to refer to a user interface that applies concepts of natural interaction in its construction [5]. NUI is considered a new interaction paradigm, since the old and known WIMP (Window, Icon, Menu, Pointer) use concepts of interaction with devices themselves, i.e., there is the use of keyboard or mouse to access any part of the graphic interface, what is not considered as natural interaction, since using such hardware is not part of the naturalness of human being. 
Telemedicine or eHealth is one area that could benefit from this kind of technology. The widespread use of computer systems in healthcare is now a reality. Systems that can connect doctors, patients and students or make possible the evaluation of diagnostic tests from distance are good examples. Trends in telemedicine are systems that are capable of adding more advanced modes of interaction, such as threedimensional interfaces and natural interaction.

\section{Interaction in Telemedicine}

It is clear from the literature [07] that the term telemedicine encompasses many different technologies, used in different ways. According to a review [07] that compares the provision of patients using face-to-face care with care using telecommunications technologies, sometimes telemedicine appliances make use of ongoing technologies or they are stimulated by enthusiasts more than clinical needs.

One example of technologies well-done applied in Telemedicine issues is the Virtual Reality. According with Gamberini (2001) the use of VE (Virtual Environment) lets users navigate and interact with a three-dimensional environment in real time. The use of VR features brought many real possibilities to Telemedicine systems, such as the remote manipulation of equipment, remote procedures training, interaction with anatomic models. This kind of feature is special useful for student training, surgery planning and also neuropsychological rehabilitation [08].

Telemedicine is a multidisciplinary field that can aid many aspects of health care, one of those is prevention. In this field, motivating factors and incentives are necessary to convince people to do sports, exercises, change nutrition type or composition and have a healthy lifestyle. This action can prevent diseases or obesity and cardio problems. To accomplish this task the serious games are an excellent opportunity, besides prevention they can also help with rehabilitation and therapy. Clinical feedback such as movement data can provide medical experts with useful information regarding patients' home training [09].

In order to use a more realistic game environment it is necessary to have some kind of equipment that provides for user a natural and transparent interaction. So that the experience is as natural as possible. In other words, we need to improve Natural Interaction (NI) features or using a Natural Interaction Interface (NUI). We have many options to develop this kind of features, different equipments and software. As a low cost alternative the Microsoft Kinect can be used as a full body input device. There are examples for telemedicine that uses NUI through Kinect, such as patient assistance and monitoring. In [10] is discussed a system that provides information about the exercises and gives feedback of the patient performance, also allowing the therapist to observe sessions remotely and recording them. Another example is discussed in [11], where the Kinect is used for improving stroke rehabilitation. This work shows different implementations using Kinect and evaluates them, showing the suitability of Kinect for being used in such application. González et al (2012) discusses the use of Kinect and Wii Balance Board as a low-cost alternative to estimate the center of mass of patients. This solution is closely related to human motion stability while walking 
and standing and it is very necessary in an increasingly older population. Otherwise, using Kinect and Wii, instead specific equipment, is a solution more accessible and portable. This solution can be also used for rehabilitation, motion stability assessment and balance training. Another telemedicine scenario that suits well with NUI through Kinect is the distance learning and training. For example, the use of NUI for better teaching-learning user interfaces. Medeiros et al (2012) presents a solution for enrich the user experience in a telemedicine system called Arthron as detailed in the next section.

\section{IN Features in Arthron}

The proposal of IN features for Arthron [18] was based in the need of a better user interface solution for 3D manipulation improved by our own experience in using Arthron for telemedicine activities. Besides, a "handless" user interface is very suitable for surgeries rooms and also telemedicine rooms.

The GTAVCS [19] developed Arthron tool which is a remote management tool for capturing and distributing multiple simultaneous streams of media to provide support for several video-collaboration scenarios, as broadcast surgeries in the context of telemedicine. The Arthron main feature is to offer the user a simple interface for handling different sources/streams of media simultaneously. Therefore the user can remotely add, remove, configure the presentation format and schedule the exhibition in time and space of media streams as shown in Figure 01-(a).

An innovation of Arthron is to provide the possibility to manipulate $3 \mathrm{D}$ objects, especially human anatomical structures, while viewing other streams, such as video. The addition of these 3D models is especially useful as a didactic resource focused to distance training and learning. Through this feature the physician-teacher can show students in an integrated manner to live video, models that demonstrate the normal organs function, tissues or structures of the human body. In Figure 01-(b) we present the integration of 3D models to Arthron tool.

(a)
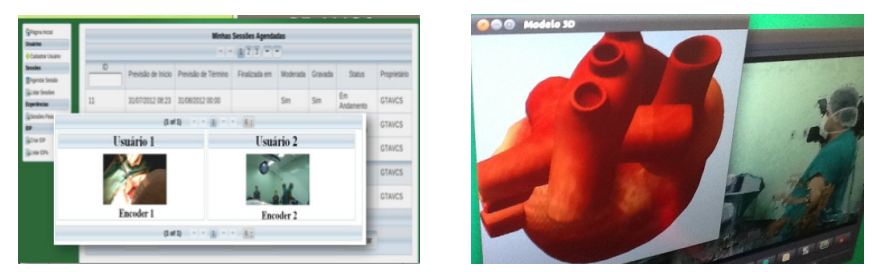

Fig. 1. Arthron User Interfaces. In (a) The interface for video manipulation and in (b) the 3D models used in a surgery transmission with Arthron.

In the 3D model manipulation section of this application was introduced the concept of natural interaction with the Kinect tool. This feature makes it easier the use and manipulation of the 3D models while viewing other flows managed by Arthron. After all in Telemedicine the using the hands and touch prevent simultaneous 
execution of medical procedures due to sterilization. In this sense the interaction without touching devices to manipulate $3 \mathrm{D}$ objects makes it possible that the physician can control and manipulate three-dimensional models during surgery.

As we mentioned before, the main goal of using IN features in Arthron was an alternative way to access the control functions used for 3D manipulation. So a gesture vocabulary was defined to identify the users modes of interaction. Figure 02 shows the main features which are used to interact with the application.

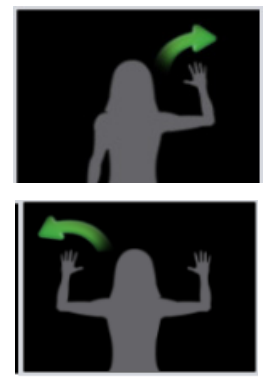

(a)

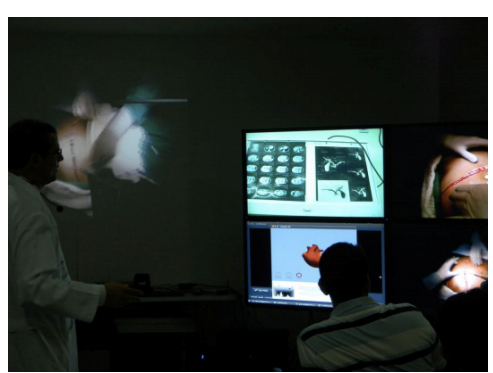

(b)

Fig. 2. IN in Arthron. In (a) gestures vocabulary, where it shows how to start the both hands tracking, starting with the left hand. In (b) interaction with Kinect, the doctor is manipulating 3D models using Kinect. In this experience we are using a video wall with multiples video streams and the 3D models are loaded together.

For development IN features in Arthron we used the Microsoft Kinect to capture the depth image stream and the framework OpenNI to assist the depth stream processing. The Tracker component recognizes gestures and begins tracking the identified hand. The Renderer component then receives the hand tracking and decides how to manipulate the 3D object. To better understand the application you need to know that it was developed in a distributed manner. Components Screen, 3D Object and Gesture are used to interface with the user, access the 3D objects and interpret gestures, respectively.

\section{Discussion}

This work was developed focusing on the benefits that the use of Natural Interaction would provide for e-Heath or Telemedicine systems improvement. The Kinect was used to add movement detection to a preexistent tridimensional application, though OpenNI framework for Kinect. The primary results of user experiences figure that telemedicine systems as a large field of research and innovation for IN features.

So, IN is a "natural" user interface and it can improve, specially for complex systems, a easier way for interaction. On the current work stage it is possible to adapt the proposed solution for others scenarios of application, as training and remote manipulation. 
Acknowledgments. We are grateful to the Lauro Wanderley Academical Hospital professionals for the partnership that made this work possible, specially Dr. Geraldo Almeida.

\section{References}

1. McCord, Charlotte. Gestures, Western Folklore 7, 290-292 (1948)

2. Frati, V.: Using Kinect for hand tracking and rendering in wearable haptics. In: World Haptics Conference (WHC) at IEEE (2011)

3. Researchers use Kinect gesture control in stroke rehab system. The Engineer (2012)

4. Hewett: Curricula for Human-Computer Interaction. The Association for Computing Machinery, Special Interest Group on Computer Human Interaction (2009), http: //old.sigchi.org/cdg/cdg2.html\#2_1

5. Vieira, E., Passos, M., Santos, B.A., Oliveira, S.S., Melo, E.A., Motta, G.H.M.B., Tavares, T.A., Filho, S., de Lemos, G.: Uma Ferramenta para Gerenciamento e Transmissão de Fluxos de Vídeo em Alta Definição para Telemedicina. Salão de Ferramentas do Simpósio Brasileiro de Redes de Computadores 2012, Ouro Preto. Anais do Salão de Ferramentas do SBRC (2012)

6. Rauterberg, G.W.M., Mauch, T., Stebler, R.: How to improve the quality of human performance with natural user interfaces as a case study for augmented reality. In: Smith, T.F., Waterman, M.S. (eds.) Identification of Common Molecular Subsequences (1996); J. Mol. Biol. 147, 195-197 (1981)

7. Currell, R., Urquhart, C., Wainwright, P., Lewis, R.: Telemedicine versus face to face patient care: effects on professional practice and health care outcomes. The Cochrane Collaboration (2010), http: / /www . thecochranelibrary.com/userfiles / ccoch/file/Telemedicine/CD002098.pdf (review)

8. Riva, G., Gamberini, L.: Virtual Reality in telemedicine. ch. 7. Communications Through Virtual Technology: Identity Community and Technology in the Internet Age (2001), http: / / www . neurovr.org/emerging/book1/1CHAPT_07.PDF

9. Wac, K., Hausheer, D., Fiedler, M., Bonato, P., et al.: Future Internet for eHealth. Report from Dagstuhl Seminar 12231 (2012),

http://publik.tuwien.ac.at/files/PubDat_210335.pdf

10. Ni, B., Pei, Y., Winkler, S.: Kinect for Rehabilitation, http://vintage.winklerbros . net/Publications / iCREATe2 012 .pdf

11. LaBelle, K. Evaluation of Kinect Joint Tracking for Clinical and In-home Stroke Rehabilitation Tools. Notre Dame, Indiana. 2011. http://www1bpt.bridgeport.edu/ jelee/courses/CS550_F12/Thesis/T3.Kinect\%20Joint.pdf

12. González, A., Hayashibe, M., Fraisse, P.: Estimation of the Center of Mass with Kinect and Wii balance board. In: IROS12: International Conference on Intelligent Robots and Systems, Portugal (2012), http: / / hal-lirmm. ccsd.cnrs.fr/docs / $00 / 73 / 46 / 29 / \mathrm{PDF} /$ IROS2012.pdf

13. Medeiros, A., Castro, R., Tavares, T.A.: Natural Interaction for 3D Manipulation in Telemedicine: A Study Case developed for Arthron Video Collaboration Tool. In: Workshop de Realidade Virtual e Aumentada, Paranavaí. Anais do WRVA 2012, vol. 1 (2012) 